\title{
The Effects of Perceptual Grouping and Category Boundary Salience on Location Memory
}

\author{
Emily K. Farran ${ }^{1 *}$, Sarah C. Connell ${ }^{2}$, Bhupinderjit K. Pharwaha ${ }^{2}$ \\ ${ }^{1}$ Department of Psychology and Human Development, Institute of Education, University of London, London, UK \\ ${ }^{2}$ School of Psychology and Clinical Language Science, University of Reading, Reading, UK \\ Email: *E.Farran@ioe.ac.uk
}

Received August $15^{\text {th }}$, 2012; revised September $14^{\text {th }}, 2012$; accepted October $12^{\text {th }}, 2012$

\begin{abstract}
The type of information used to process spatial layouts was assessed by observing the effect of spatial category salience and perceptual grouping (a non-spatial category), on a location memory task. Participants $(\mathrm{N}=64)$ learnt the pairings between twenty objects and twenty marked locations within a "house". They then placed the objects in the remembered locations, without the aid of location markers. Spatial category salience was manipulated by presenting the house as an open space (no boundary condition) or by dividing the space into quadrants (boundary condition). Perceptual grouping was manipulated by using identical shapes (control condition) or sets of shapes which identified triads of objects (perceptual grouping condition). Both non-spatial and spatial categories improved location memory accuracy. The nonspatial category produced a prototype effect and the spatial category produced a subdivision effect. Different patterns of category dominance (spatial vs. non-spatial) were observed for level of accuracy compared to distortion effects.
\end{abstract}

Keywords: Spatial Category; Perceptual Grouping; Memory; Prototype Effect; Subdivision Effect

\section{Introduction}

The concept of a "location" is innately relative; it is described in reference to elements such as other objects (Hund \& Plumert, 2003), landmarks (Sadalla, Burroughs, \& Staplin, 1980) and regions (Plumert \& Hund, 2001). The Category Adjustment (CA) model (Huttenlocher, Hedges, \& Duncan, 1991) was put forward to explain how individuals encode spatial location information and retrieve this information from memory. According to this model, spatial location coding involves two steps. Individuals first estimate location using fine-grained information, i.e. the distance and direction of an object's location from a referent. Second, estimates are adjusted using categorical information about region membership. Each category is represented by a prototype at the centre of the region; adjustments are made towards the prototype.

The weightings of fine-grained and categorical information are dependent on the degree of uncertainty over fine-grained information. Inexactly represented stimuli are adjusted in accordance with category membership. This enhances the accuracy of a location estimate, but also introduces distortions. For example, individuals overestimate or underestimate distances between objects that are in different regions or the same regions respectively; the subdivision effect (Huttenlocher et al., 1991).

Plumert and Hund (2001) investigated the subdivision effect in 7-, 9- and 11-year-olds and adults. Participants learnt 20 locations within a 32 inch $^{2}$ box. Hedges, Corrigan and Crawford (2004) report that space is naturally portioned into regions divided about the cardinal axes. Plumert and Hund (2001) exploited this by increasing the salience of the quadrants of the square box across three conditions: the box either had no boundaries present, or was divided into quadrants by lines or by

\footnotetext{
"Corresponding author.
}

walls. This increased spatial category salience, but also provided more spatial referents (i.e. lines/walls), another factor which is known to improve accuracy and prototype formation (Fitting, Wedell, \& Allen, 2005). Results showed that, for all groups, boundary salience improved accuracy. All groups overestimated between quadrant distances. Significantly longer estimates were observed for between than within quadrant distances for the 11-year-olds and adults only, indicative of a subdivision effect in these groups.

There is much support for the use of spatial categories as a method of adjusting location estimates, but little research relating to non-spatial categories. Huttenlocher, Hedges and Vevea (2000) presented participants with stimuli which varied along a spatial (fish fatness) or non-spatial dimension (square greyness). On removal of each stimulus exemplar, participants were asked to reproduce it. Biases in estimations were dictated by the distribution of the exemplars. The pattern of results was similar across spatial and nonspatial categories, which suggests that the CA model is not restricted to spatial categories.

Hund and Plumert (2003) used a similar experimental space to Plumert and Hund (2001), but the objects employed could be categorised using non-spatial information (e.g. vehicles, animals). In a "related" condition, items were placed such that objects belonging to the same non-spatial category were in the same spatial category (same quadrant). In the "unrelated" condition, object-location pairings were random. In the related condition only, children and adults underestimated the distances between objects that belonged to the same non-spatial category, i.e. a prototype effect. This suggests that non-spatial information can bias estimates of spatial locations. However, as spatial and non-spatial categories were congruent one cannot determine whether the bias observed was due to an amplifying effect of the non-spatial categories on spatial category representation, 
or whether it was independent of spatial categories.

It appears that, at least in terms of distortions, a similar effect occurs for non-spatial categories, as observed for spatial categories. However, Hund and Plumert (2003) did not demonstrate any effects of non-spatial categories on location accuracy. This might be indicative of a less powerful influence of non-spatial categories on location memory than spatial categories. This cannot be determined from their experimental design.

Consideration has not been given to how two or more distorting elements interact with one another, as they inevitably would in real-world spaces. In this experiment, the relative influence of spatial category salience and a non-spatial factor, perceptual grouping, on location memory will be determined. Perceptual grouping is the grouping together of elements into a global whole, based on shared properties such as luminance or shape (Wertheimer, 1923). It has some parallels with the physical biases observed in location memory; perceptual grouping is a perceptual effect based on shared perceptual properties, whilst location biases induce physical grouping based on shared spatial properties.

Perceptual grouping can have an effect on the perceived spatial characteristics of items. Coren and Girgus (1980) presented participants with four aligned elements that perceptually grouped into pairs, e.g. two black and two white equally spaced circles (luminance grouping). Participants reported within (e.g. black-black) and between (e.g. black-white) perceptual groups as closer together and further apart than they really are respectively.

Hommel et al. (2000) showed participants a projected image of 18 "houses", grouped by colour or shape. These were arranged in an approximate oblique four by four formation of four sets of four items, with two peripheral distracter items. Interestingly, although they showed an effect of perceptual grouping for response times to a location judgement task ("Is X above Y?”), there was no effect of perceptual grouping on distance estimates. However, the response map was smaller than the projected image (ratio, 1:2), which might have reduced any affect of distance estimates. In addition, due to the structured arrangement, objects were grouped by good continuation, which could have dictated distance estimates.

The current study, based on Plumert \& Hund (2001), investigated the role of perceptual and spatial factors on location memory. Category salience was manipulated by dividing the experimental space using physical boundaries. Target items could be perceptually grouped by shape similarity. In contrast to Hund and Plumert (2003), non-spatial categories (in this case, shapes) crossed spatial category boundaries. This incongruence enabled us to determine separable influences of perceptual grouping and spatial category salience on location memory, and how these two distorting factors interact. Based on the CA model, it is predicted that increased spatial category salience will improve accuracy and induce a subdivision effect. If perceptual grouping, a non-spatial category, provides an appropriate referent, then accuracy will also be improved for grouped compared to control objects, and a prototype effect should be observed towards the centre of perceptual groups. Both spatial and non-spatial categories should provoke underestimated within quadrant distance estimates. Between quadrant distance estimates should be overestimated if spatial category salience is a stronger referent, or underestimated if perceptual grouping is the stronger referent to location memory.

\section{Method}

\section{Participants}

Sixty-four participants from the University of Reading, with mean (S.D.) age: 20 years, 4 months (3 years, 0 month) took part. Participants had normal/corrected to normal vision.

\section{Materials}

An open box, referred to as a "house", was employed (measurements: 32 inches [width] $\times 32$ inches [depth] $\times 13$ inches [height]). The house could be divided into four equal quadrants (16 inches ${ }^{2}$ ) by inserting 13 inch high opaque walls. The walls were in place for "boundary" trials only. The base consisted of a layer of Perspex above a layer of MDF, separated by 0.5 inch removable floors (32 inches ${ }^{2}$ ) could be inserted between the Perspex and MDF. This ensured that the floor could be changed without disrupting the location of any objects that had been placed on the Perspex. There were two training floors, one for control trials and one for perceptual grouping trials (Figures 1(a) and (b)). Each depicted 20 to-be-remembered locations as black dots (dot area: 0.3 inch $^{2}$ ). Twelve of these dots were arranged into four "target triads" of 3 dots that were each 6 inches from one another (equilateral triangle formation). If one considers the house in terms of four quadrants, then each target triad straddled a quadrant boundary, such that two dots were in the same quadrant of the house, and the third was in an adjacent quadrant. Each quadrant also featured two of the remaining eight "distracter" dots. At test, a plain white floor was employed. To record the locations of placed objects, a measurement floor was inserted. This was a grid of vertical and horizontal lines, spaced at 0.5 inch intervals.

Each participant took part in a control condition and a perceptual grouping condition. These differed in terms of the set of objects that were employed. In both conditions, objects were 20 different coloured (different for each set: 40 colours in total) two-dimensional shapes with an area of 2 inches $^{2}$ and a hole through their centre. The objects employed in the control condition were all the same, capsule, shape (Figure 1(a)). The objects employed in the perceptual grouping condition comprised of 8 shape types. The target triad locations were paired with 3 hearts, 3 squares, 3 circles and 3Xs. To explain, within each target triad the 3 items were the same shape, and the shape type differed across target triads. The remaining shapes were 2 kites, 2 Hs, 2 triangles and 2 stars. These were paired with the eight distracter locations (Figure 1(b)).

\section{Design \& Procedure}

Participants were randomly assigned to either boundary (house divided into quadrants by opaque walls) or no boundary (house not divided) conditions (between-participant factor) and each participant took part in a control and a perceptual grouping condition (within-participant factor). Thus, there were two independent variables; boundary condition (boundary, no boundary); and perceptual grouping condition (perceptual grouping, control). The control condition was carried out first, followed by the perceptual grouping condition. This was designed to eliminate the possibility of carryover effects from the perceptual grouping condition to the control condition, i.e. by differentiating target triads in the perceptual grouping condition, participants might have been more likely to notice target triads 


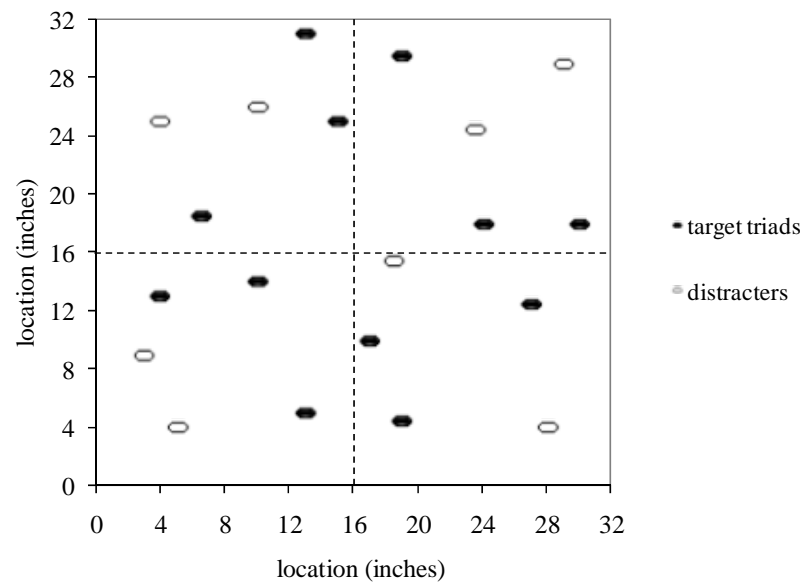

(a)

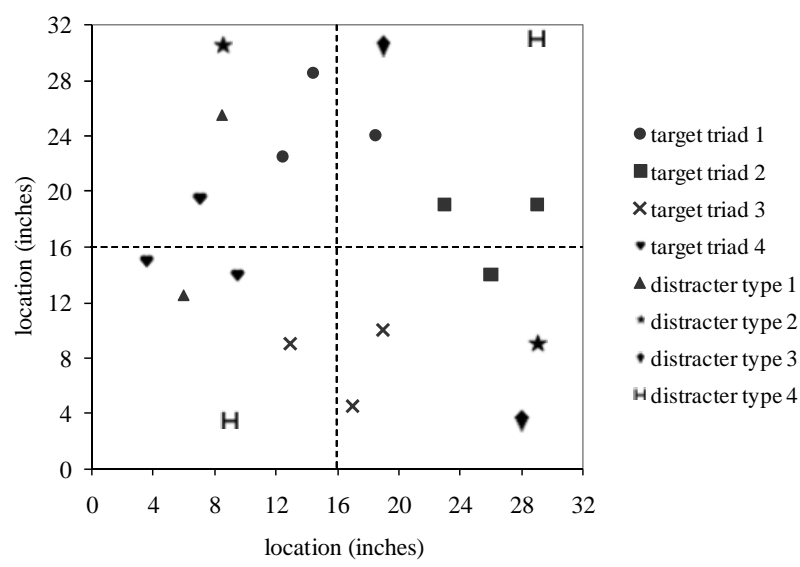

(b)

Figure 1.

(a) Object locations and shapes employed for the control conditions (shapes not drawn to proportion). Note, shapes are shown as filled or unfilled to differentiate target from distracter items. In the experiment, shapes were individually differentiated using twenty different colours; (b) Object locations and shapes employed for the perceptual grouping conditions (shapes not drawn to proportion). Note, all shapes are shown as black. In the experiment, shapes were individually differentiated using twenty different colours.

in the control condition (Although, note that Verbeek \& Spetch, 2008 report in their location memory study that the order of control and experimental conditions did not affect performance).

Each condition comprised of a training phase followed by a single test trial. In the training phase, a training floor was in place throughout. Participants watched the experimenter place the 20 objects on the 20 location dots and were instructed to remember the object-location pairings. Circular holes in the centre of each object permitted visibility of the location dots. Once all 20 objects had been placed by the experimenter, the participant was asked to turn around so that the house was no longer in view. The experimenter removed the twenty objects. The participant faced the house again, was handed each object in turn (random order) and was asked to place it on the correct location dot. For each object, if the participants placed it on the correct location dot, they were handed the next object. If the participant placed the object on the incorrect location dot, the experimenter informed them of their error and moved it to the correct location dot before handing them the next object. Training trials were repeated until the participant completed a training trial in which they placed each of the 20 objects on the correct location dot without error (i.e. the experimenter made no corrections). This determined that the participant had memorised the object-location pairings. The training floor was then removed and replaced by test floor. In the test trial participants were asked to place the objects in the correct locations onto the plain white floor, this time using their memory of the precise location of each of the 20 objects.

After completion of the test trial, the test floor was removed (the objects remained on the clear Perspex) and the measurement floor was inserted and the $\mathrm{x}$ and $\mathrm{y}$ coordinates of each of the 20 object placements was measured to the nearest 0.5 inch. A number of dependent variables were derived, and are explained fully in the results section. In the training phase, the dependent variable was the number of training trials required to reach criterion. In the test phase, the dependent variables were accuracy score, triad central displacement score and between and within quadrant distance estimate score.

\section{Results}

All data was analysed using statistics software: Statistical Package for the Social Sciences (SPSS).

\section{Training Phase}

The mean (S.D.) number of training trials required to reach criterion was: boundary, perceptual grouping condition: 4.13 (1.61) trials; boundary, control condition: 6.19 (2.29) trials; no boundary, perceptual grouping condition: 4.59 (2.17) trials; no boundary, control condition: 6.78 (3.58) trials. ANOVA was carried out on the number of training trials, with a betweenparticipant factor of boundary (boundary, no boundary) and a within-participant factor of perceptual grouping (control, perceptual grouping). This showed a main effect of perceptual grouping, $F(1,62)=61.42, p<0.001$ (control $>$ perceptual grouping). There was no main effect of boundary or boundary by perceptual grouping interaction ( $F<1$ for both).

\section{Test Phase}

Despite demonstrating competency in the training phase, some object-location pairings were incorrect at test. Some participants transposed the locations of two objects of similar colours or, in the perceptual grouping condition, two objects of identical shape. Consistent with similar studies (Hund \& Plumert, 2003), transpositions were counted as accurate placements. If transpositions could not be accounted for by object similarity, they were counted as errors and excluded. Transpositions and errors were rare; control conditions: $1.6 \%$ transpositions, $0.4 \%$ errors; perceptual grouping conditions: $0.5 \%$ transpositions, $0.7 \%$ errors.

\section{Accuracy Scores}

Accuracy scores measure displacement (inches) between each observed object placement location at test and the true location of the object. The accuracy score for each individual is a mean of their displacement distances across all 20 objects for that condition. Lower scores indicate higher accuracy $(0=$ 
100\% accuracy) (Figure 2). Accuracy scores were analysed using ANOVA with a between-participant factor of boundary (boundary, no boundary) and a within-participant factor of perceptual grouping (control, perceptual grouping). There was a significant main effect of perceptual grouping, $F(1,62)=4.07, p=$ 0.05 , due to higher accuracy in the perceptual grouping than control conditions. There was a main effect of boundary, $F(1,62)=$ $12.57, p=0.001$, due to higher accuracy in the boundary than no boundary conditions. Although marginal, a boundary by perceptual grouping interaction $(F(1,62)=3.27, p=0.08$ ) was explored to reveal that despite an effect of boundary for both control $(t(62)=4.03, p<0.001)$ and perceptual grouping conditions $(t(62)=2.02, p=0.05)$ the main effect of perceptual grouping was driven by the no boundary condition (no boundary: $t(31)=2.48, p=0.02$; boundary: $t(31)=0.16, p=0.87$ ).

\section{Triad Central Displacement Score: A Measure of Non-Spatial Categorical Coding}

Triad central displacement scores measured the extent to which observed object placements for target triad items at test were clustered towards each triad centre (determined according to the true locations); the true distance between each object and the triad centre was subtracted from the observed distance between each object and the triad centre. Positive and negative scores indicate a displacement away from or towards the triad centre respectively (Figure 3). The triad displacement score for each individual is a mean of their displacements for the target triad locations only (12 locations).

ANOVA was carried out with a between-participant factor of boundary (boundary, no boundary) and a within-participant factor of perceptual grouping (control, perceptual grouping). This showed a main effect of perceptual grouping $F(1,62)=$ $71.59, p<0.001$; objects were placed closer to the triad centres in the perceptual grouping, than the control conditions. One sample t-tests compared to zero (100\% accuracy) revealed that objects in the perceptual grouping conditions, were grouped together towards the triad centres $(t(63)=-4.34, p<0.001)$. Objects in the control condition were not grouped together, they were placed significantly further away from the triad centres than the true locations $(t(63)=7.81, p<0.001)$. There was no effect of boundary $(F(1,62)=2.56, p=0.12)$ or perceptual grouping by boundary interaction $(F<1)$.

\section{Between and within Quadrant Distance Estimate Scores: A Measure of Spatial Categorical Coding}

Each target triad crossed a quadrant boundary; two objects were within the same quadrant, and the third was in the adjacent quadrant. Actual between and within quadrant distances between pairs of objects in the triad were all 6 inches. Using target triad locations only, difference scores were created between each observed and true between and within quadrant distance, and means created. Positive scores and negative scores indicate overestimated and underestimated distances respectively (Figure 4).

Distance estimate scores were analysed using ANOVA with a between-participant factor of boundary (boundary, no boundary) and within-participant factors of perceptual grouping (control, perceptual grouping) and distance estimate (within quadrant, between quadrant). There was a main effect of perceptual grouping, $F(1,62)=71.63, p<0.001$ (perceptual grouping

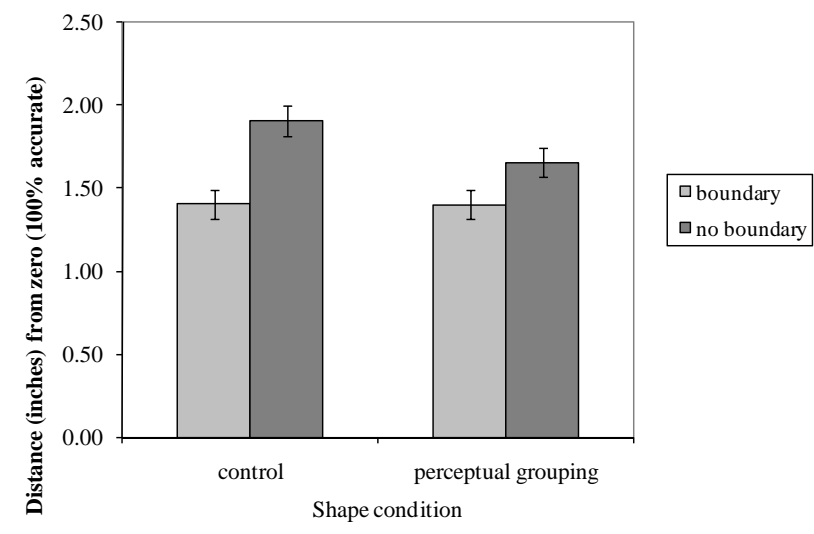

Figure 2.

Accuracy scores: mean (S.D.). Accuracy score shows distances in inches from actual location (a lower score indicates higher accuracy).

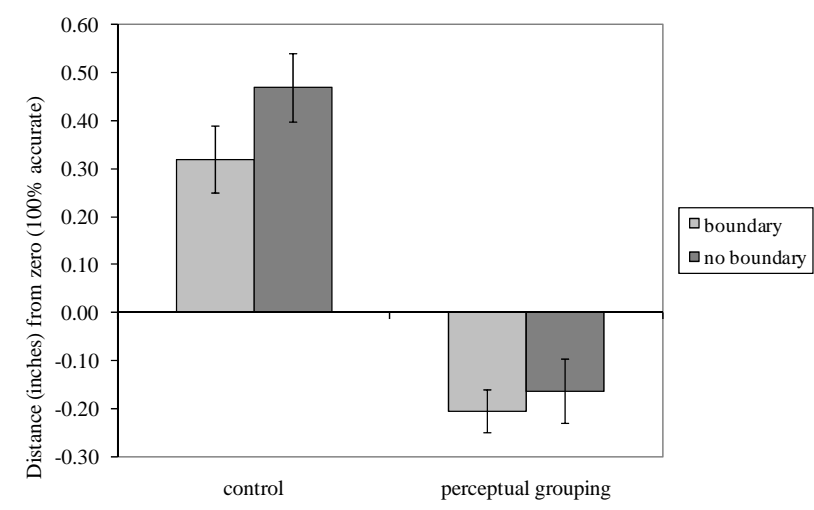

Figure 3.

Triad central displacement scores: mean (S.D.). Positive and negative scores indicate displacement away from and towards the triad centre.

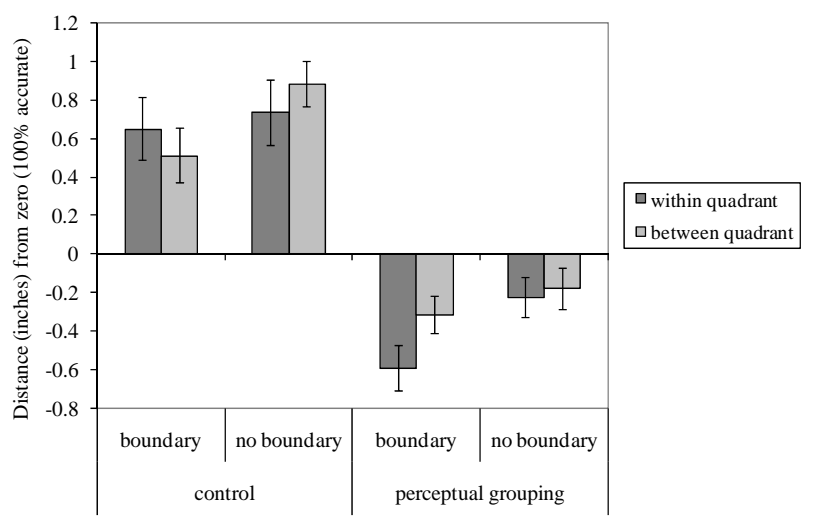

Figure 4.

Within and between quadrant distance estimate scores: mean (S.D.). Positive and negative scores indicate overestimated and underestimated distances.

score $<$ control score). Separate one sample t-tests compared to zero (100\% accuracy) demonstrated overestimated distances in the control condition $(t(63)=7.55, p<0.001)$ and underestimated distances in the perceptual grouping $(t(63)=-4.53, p<0.001)$. There was a main effect of boundary, $F(1,62)=4.67, p=0.04$ (no boundary score $>$ boundary score). One sample t-tests against zero (100\% accuracy) showed accurate responses on the 
boundary condition $(t(31)=0.87, p=0.39)$, but significant overestimates in the no boundary condition $(t(31)=3.62, p=0.001)$. There was no effect of distance estimate $(F<1)$ and no two way interactions (distance estimate by boundary, $F<1$; perceptual grouping by boundary, $F<1$; distance estimate by perceptual grouping, $F(1,62)=2.35, p=0.13)$. There was a significant three-way interaction between perceptual grouping, distance estimate and boundary, $F(1,62)=6.18, p=0.02$. Exploration of this revealed a boundary by distance estimate interaction for the perceptual grouping condition only (control condition: $F(1,31)=1.75, p=0.19$; perceptual grouping condition: $F(1,31)$ $=5.54, p=0.02$ ) on account of significantly lower within than between distance estimates in the boundary condition only (boundary: $t(31)=-3.77, p=0.001$; no boundary: $t(31)=-0.72$, $p=0.48)$. Although, note that both within and between quadrant distances were underestimated (within: $t(31)=-5.15, p<$ 0.001 ; between: $t(31)=-3.19, p=0.003)$.

\section{Discussion}

As predicted by the CA model, and consistent with previous research (Plumert \& Hund, 2001), spatial category salience had a positive effect on accuracy, and also showed evidence of systematic biases on location memory responses. Importantly, a similar effect of improved accuracy, and systematic distortions was also observed for a non-spatial factor, perceptual grouping. Thus, spatial and non-spatial categorical information influence spatial estimation. Accuracy and bias are considered separately below.

Spatial and non-spatial categories had separate effects on location memory accuracy. Although marginal, the interaction between these two factors suggests a dominance of one factor over the other; although spatial category salience influenced accuracy regardless of the perceptual grouping condition, an effect of perceptual grouping on accuracy was observed when spatial category salience was low (no boundary condition), but not when it was high (boundary condition). This demonstrates that locations can be coded with reference to more than one factor or category, but that improvement to location memory accuracy is primarily dictated by the strongest available referent. In this case, high spatial category salience was a stronger referent than perceptual grouping.

The biases induced by perceptual grouping and spatial categories on location memory revealed a different pattern to the accuracy data. When both categories were present (i.e. the perceptual grouping, boundary condition) concurrent distorting effects were observed; a prototype effect of perceptual grouping and a subdivision effect of spatial category salience. Thus, more than one factor can influence location memory at any one time. This finding is important as it contributes to our understanding of real-world location coding, where multiple perceptual and spatial referents are present.

The study was designed such that perceptual grouping and spatial categories were incongruent. Thus, although additive effects of accuracy could be observed, any distorting effects of each factor could not, by design, be additive. This enabled us to determine the relative influence of each factor. Both factors distorted responses in the predicted directions; however this was stronger for perceptual grouping than for spatial category salience. Perceptual grouping distorted responses towards the centre of each perceptual group, i.e. a prototype effect as predicted. This occurred universally, irrespective of spatial cate- gory salience. A subdivision effect was predicted in relation to spatial categories. This was observed in the boundary condition, but only for the perceptual grouping condition. In this condition, the competing effects of perceptual grouping and spatial category salience can be observed. Both between and within distances were underestimated in line with a perceptual grouping prototype effect, but this was less strong for between than within quadrant distances, i.e. the pattern dictated by a subdivision effect across spatial categories. Thus, when perceptual grouping and spatial category salience compete, the overriding distorting influence originates from perceptual grouping.

Just as bias effects index categorical coding, performance on the training trials is also a measure of categorical encoding. This is because fine-grained information is provided by the training dots. The pattern of performance in the training phase showed that fewer training trials were required for the perceptual grouping than control conditions to reach criterion, whilst no difference was observed between boundary and no boundary conditions. This again suggests that, for categorical coding, perceptual grouping dominates over spatial category salience.

One cannot discuss the bias effects of spatial category salience in the perceptual grouping conditions, without alluding to the lack of effect in the control condition. The control condition is similar to Plumert and Hund (2001), i.e. spatial category salience is the only influencing factor on performance. Thus, one would predict distortion effects akin to Plumert and Hund (2001). The lack of effect in the control condition could relate to the objects employed. Whilst previous studies used easily nameable and differentiable objects, the objects used here, by design, could only be differentiated by colour. Although errors and transpositions were low, perhaps this influenced the way in which object-location pairings were remembered. In contrast, an effect of spatial category salience was observed when shapes were perceptually grouped. Perhaps perceptual grouping brought attention to the target triads, thus emphasising that these groups crossed category boundaries, and in turn introducing an effect of category salience.

The present results show a consistent pattern of biases to Hund and Plumert (2003) who also report a prototype effect towards the centre of groups of non-spatially related objects. By using incongruent spatial and non-spatial categories, the present study further qualifies this effect; we have shown that the prototype effect is not related to spatial category. The present results also support our previous assertion relating to Hommel et al. (2000). We suggested that they failed to show distortions in relation to perceptual groups on account of design confounds. We have shown that, when these confounds are eliminated, grouping by shape similarity has a distorting effect on object placements. We cannot, therefore, support Hommel's et al. (2000) notion that the organisation of spatial information is not assessed by distance estimation.

Although Hund and Plumert (2003) report similar biases to those observed here, they did not find an effect of non-spatial categories on accuracy, even when spatial categories were not made salient (equivalent to the "no boundary" condition). This contrasts to the present results, where accuracy was affected by non-spatial categories (perceptual grouping) in the no boundary condition. This difference across studies can be explained by the nature of each non-spatial category. In Hund and Plumert (2003) the objects were related by function. Functional categories require higher level processing than perceptual grouping, a low-level preattentive process (Treisman, 1982). We suggest 
that, in the present study, perceptual grouping categories were encoded preattentively, which influenced subsequent location coding. In contrast, object function might be coded in parallel to object locations. It appears then that, although object function has a distorting effect on location memory, it has a minimal impact on location memory accuracy.

The present results support the CA model in terms of the biases and effects on accuracy in relation to both spatial and nonspatial categories. Analyses of accuracy demonstrated a dominance of spatial categories over non-spatial categories. Analyses of biases, on the other hand, showed that between quadrant distance estimates were underestimated when both categories were present, indicative of a stronger distorting influence from perceptual grouping than spatial categories. Thus, spatial and non-spatial categories can both influence location memory at any one time, but the interplay between such categories differs according to the resulting biases or the effects on accuracy.

\section{REFERENCES}

Coren, S., \& Girgus, J. S. (1980). Principles of perceptual organization and spatial distortion-The Gestalt illusions. Journal of Experimental Psychology: Human Perception and Performance, 6, 404-412. doi:10.1037/0096-1523.6.3.404

Fitting, S., Wedell, D. H., \& Allen, G. L. (2005). Memory for spatial location: Influences of environmental cues and task field rotation. In A. G. Cohn, \& D. M. Mark (Eds.), Spatial information theory: International Conference (pp. 459-474). Berlin: Springer-Verlag.

Hirtle, S. C., \& Kallman, H. J. (1988). Memory for the locations of pictures: Evidence for hierarchical clustering. American Journal of Psychology, 101, 159-170. doi:10.2307/1422832

Hommel, B., Gehrke, J., \& Knuf, L. (2000). Hierarchical coding in the perception and memory of spatial layouts. Psychological Research,
64, 1-10. doi: $10.1007 / \mathrm{s} 004260000032$

Hund, A. M., \& Plumert, J. M. (2003). Does Information about what things are influence children's memory for where things are? Developmental Psychology, 39, 939-948. doi:10.1037/0012-1649.39.6.939

Huttenlocher, J., Hedges, L. V., \& Duncan, S. (1991). Categories and particulars: Prototype effects in estimating spatial location. Psychological Review, 98, 352-376. doi:10.1037/0033-295X.98.3.352

Huttenlocher, J., Hedges, L. V., \& Vevea, J. L. (2000). Why do categories affect stimulus judgement? Journal of Experimental Psychology: General, 129, 220-241. doi:10.1037/0096-3445.129.2.220

Newcombe, N. S., Huttenlocher, J., Drummery, A. B., \& Wiley, J. G. (1998). The development of spatial location coding: Place learning and dead reckoning in the second and third years. Cognitive Development, 13, 185-200. doi:10.1016/S0885-2014(98)90038-7

Plumert, J. M., \& Hund, A. M., (2001) The development of memory for locations: What role do spatial prototypes play? Child Development, 72, 370-384. doi:10.1111/1467-8624.00284

Sadalla, E. K., Burroughs, W. J., \& Staplin, L. J. (1980). Reference points in spatial cognition. Journal of Experimental Psychology: Human Learning and Memory, 6, 516-528. doi:10.1037/0278-7393.6.5.516

Schmidt, T., Werner, S., \& Diedrichsen, J. (2003). Spatial distortions induced by multiple visual landmarks: How local distortions combine to produce complex distortion patterns. Perception and Psychophysics, 65, 861-873. doi:10.3758/BF03194820

Treisman, A. (1982). Perceptual grouping and attention in visual search for features and for objects. Journal of Experimental Psychology: Human Perception and Performance, 8, 194-214. doi:10.1037/0096-1523.8.2.194

Verbeek, E., \& Spetch, M. (2008). Distortions in location memory. Psychonomic Bulletin and Review, 15, 328-336. doi:10.3758/PBR.15.2.328

Wertheimer, M. (1923). First published as Untersuchungen zur Lehre von der Gestalt II. Psycologische Forschung, 4, 301-350. 